\title{
Diagnosing Wilson's Disease under the sword of Damocles
}

\author{
Ronald F. Pfeiffer \\ Oregon Health and Science University, Portland, Oregon, United States
}

\begin{abstract}
Introduction. Antos et al. [7] have reported a case of suspected uniparental disomy leading to an initial erroneous diagnosis of Wilson's Disease on the basis of genetic testing. They discuss the usefulness of the ${ }^{64} \mathrm{Cu}$ radioactive copper incorporation test as an often-overlooked diagnostic aid.

Clinical reflections. Wilson's Disease is difficult to diagnose because of its rarity, diverse clinical presentations, and the absence of a single fail-safe diagnostic test. The identification of mutations in the ATP7B gene has been an invaluable aid in the diagnosis, but genetic testing alone is not infallible, and should not be used as the sole diagnostic test in arriving at a diagnosis of Wilson's Disease.

Clinical implications. The diagnosis of Wilson's Disease must be based on a combination of findings that includes clinical history, clinical examination, and diagnostic testing. Genetic testing alone is insufficient.

(Neurol Neurochir Pol 2020; 54 (5): 364-365)
\end{abstract}

For many neurologists (myself included), the fear of missing a diagnosis of Wilson's Disease (WD) has always skulked, like a sinister spectre, in the shadowy subconscious of our neurologist brains.

This fear is based partly on the rarity of WD, with its often reported prevalence of approximately 1:30,000 (although higher in some regions) [1,2], which has fostered a propensity for WD to be diagnostically 'out of sight, out of mind.' Furthermore, its ability to masquerade as diverse disorders due to its manifold clinical manifestations ranging from hepatic to neurological to psychiatric to a plethora of other presentations [3] has seemed often to render the diagnosis of WD an enigma worthy of Elgar.

To make matters worse, there has been no easy or certain diagnostic test for WD. Screening with a serum ceruloplasmin level was simple, but neither sensitive nor specific enough to be relied upon as a sole diagnostic test [4]. A 24-hour urine copper determination was a better, though still imperfect, option, but was annoying and cumbersome for patients to collect, and was therefore often incompletely performed.

Other tests, such as liver function tests and MRI, also were not sufficiently specific. The presence or absence of the characteristic Kayser-Fleischer rings also was not a fail-safe indication of WD, since individuals with only hepatic manifestations may not have yet developed this ophthalmological
WD hallmark [5, 6]. Besides, Kayser-Fleischer rings are in fact not absolutely pathognomonic for WD [3]. Liver biopsy could be diagnostic, but entailed some risk, since coagulopathy often accompanied impaired liver function. Finally, and perhaps most dauntingly, the presence of effective treatment that could adequately control the disorder meant that missing, or even just delaying, the diagnosis could have tragic consequences.

But then the discovery that a mutation within the $A T P 7 B$ gene is responsible for $\mathrm{WD}$, and the ability to perform genetic testing for this mutation, arrived on the scene. Although initially very expensive and not readily available, with time both of these barriers were significantly overcome. The subsequent documentation of many mutations - now numbering more than 700 [2] - complicated the testing and interpretation of the results, but still it appeared that the holy grail for the diagnosis of WD had been discovered.

The report by Antos et al. [7], however, serves as an important wake-up call and reminds us that there are some holes in our holy grail. It is accepted that genetic testing may not yet have identified all mutations responsible for WD. But the reverse, that genetic testing may actually falsely identify WD, has not been widely recognised. Antos et al. now provide us with an example. The possibility of uniparental isodisomy [8], the presence of two pathogenic mutations occurring on one allele, rather than arising from the usual two alleles, leading 
to a mistaken diagnosis of WD (rather than a diagnosis of being a carrier), and subsequent inappropriate treatment, is not something that would come to the mind of most clinicians.

But it did to Antos et al., and they have alerted the rest of us to this possibility. This is especially important given that some of the treatments employed in $\mathrm{WD}$, such as penicillamine and trientine, possess the potential for serious and even life-threatening adverse effects $[2,3]$.

So, how does one avoid this potentially devastating error? Antos et al. go on to provide us with a possible answer in the form of the ${ }^{64} \mathrm{Cu}$ radioactive copper incorporation test. This is not a new test [9] but has been used rarely by most clinicians, partly because of difficulty with access to the isotope, and partly due to the complex nature of the test, which extends over multiple days.

The lesson to be learned here is not that the radioactive copper incorporation test needs to be performed on every individual suspected of having WD; that would be both impractical and unnecessary. However, in individuals in whom genetic testing suggests a diagnosis of WD but where the clinical and laboratory picture is not entirely consistent or raises some questions, this is a test that should not be forgotten.

It should be noted that the case presented by Antos et al. has some strings unfortunately left untied. The clinical picture was especially complicated and confusing, with additional comorbid diagnoses of idiopathic immunodeficiency syndrome, coeliac disease, and ulcerative colitis. Genetic testing of both parents to confirm the presence of two mutations (presumably on one allele) in one of the parents could have been confirmatory, but was impossible since the father was unknown and the mother apparently unavailable for testing. Liver biopsy during life was not performed because of concerns regarding coagulopathy, and autopsy was not performed following death, so a more secure diagnosis for the liver disease was not possible.

As noted by the authors, this case underlines that genetic testing alone should not be used as the sole criterion for the diagnosis of WD, and that an appropriate combination of clinical history, clinical examination, and laboratory findings, including genetic testing if needed, remains necessary for the accurate diagnosis of WD. This very important point is one that has also been made and emphasised by others $[10,11]$.

However, Antos et al. have brought to our attention a particularly important note of caution: although genetic testing may significantly reduce the risk of overlooking a diagnosis of WD, it also can impose a risk of incorrectly diagnosing WD.

In summary, this case presentation is a helpful and instructive lesson that will strengthen the horsehair that secures the sword of Damocles dangling above us with regard to the diagnosis - or misdiagnosis - of WD.

\section{References}

1. Hedera P. Wilson's disease: A master of disguise. Parkinsonism Relat Disord. 2019; 59: 140-145, doi: 10.1016/j.parkreldis.2019.02.016, indexed in Pubmed: 30797706.

2. Członkowska A, Litwin T, Dusek P, et al. Wilson disease. Nat Rev Dis Primers. 2018; 4(1): 21, doi: 10.1038/s41572-018-0018-3, indexed in Pubmed: 30190489.

3. Pfeiffer R. Wilson's disease. Handbook of Clinical Neurology. 2011: 681-709, doi: 10.1016/b978-0-444-52014-2.00049-5.

4. Reeve JLv, Frayling IM, Twomey PJ. Challenges in molecular diagnosis of Wilson disease. J Clin Pathol. 2020; 73(4): 181-182, doi: 10.1136/jclinpath-2019-206215, indexed in Pubmed: 32060076.

5. Taly AB, Meenakshi-Sundaram S, Sinha S, et al. Wilson disease: description of 282 patients evaluated over 3 decades. Medicine (Baltimore). 2007; 86(2): 112-121, doi: 10.1097/MD.0b013e318045a00e, indexed in Pubmed: 17435591.

6. Roberts EA, Schilsky ML. American Association for Study of Liver Diseases (AASLD). Diagnosis and treatment of Wilson disease: an update. Hepatology. 2008; 47(6): 2089-2111, doi: 10.1002/hep.22261, indexed in Pubmed: 18506894.

7. Antos A, Litwin T, Skowrońska M, et al. Pitfalls in diagnosing Wilson's Disease by genetic testing alone: the case of a 47 -year-old woman with two pathogenic variants of the ATP7B gene. Neurol Neurochir Pol. 2020; 54 (5): 478-480), doi: 10.5603/PJNNS.a2020.0063, indexed in Pubmed: 32808274.

8. Lo C, Bandmann O. Epidemiology and introduction to the clinical presentation of Wilson disease. Handb Clin Neurol. 2017; 142: 7-17, doi: 10.1016/B978-0-444-63625-6.00002-1, indexed in Pubmed: 28433111.

9. Sternlieb I, Scheinberg I. The Role of Radiocopper in the Diagnosis of Wilson's Disease. Gastroenterology. 1979; 77(1): 138-142, doi: 10.1016/s0016-5085(79)80024-4.

10. Stättermayer AF, Entenmann A, Gschwantler M, et al. The dilemma to diagnose Wilson disease by genetic testing alone. Eur J Clin Invest. 2019; 49(8): e13147, doi: 10.1111/eci.13147, indexed in Pubmed: 31169307.

11. Espinós C, Ferenci P. Are the new genetic tools for diagnosis of Wilson disease helpful in clinical practice? JHEP Rep. 2020; 2(4): 100114, doi: 10.1016/j.jhepr.2020.100114, indexed in Pubmed: 32613181. 